\title{
Exploring 3D-Virtual Learning Environments with Adaptive Repetitions
}

\author{
${ }^{1}$ Aftab Alam, ${ }^{2}$ Sehat Ullah, ${ }^{3}$ Numan Ali \\ Department of Computer Science \& IT, University of Malakand, Chakdara K.P.K, Pakistan \\ E-mail: 'alam@uom.edu.pk, ${ }^{2}$ sehatullah@uom.edu.pk, ${ }^{3}$ numan@uom.edu.pk
}

\begin{abstract}
In spatial tasks, the use of cognitive aids reduce mental load and therefore being appealing to trainers and trainees. However, these aids can act as shortcuts and prevents the trainees from active exploration which is necessary to perform the task independently in non-supervised environment. In this paper we used adaptive repetition as control strategy to explore the 3D-Virtual Learning environments. The proposed approach enables the trainee to get the benefits of cognitive support while at the same time he is actively involved in the learning process. Experimental results show the effectiveness of the proposed approach.

Keywords: Virtual Environments, Adaptive 3D-Virtual Learning environments, Cognitive aids, Multimodal feedback, Multimodal Virtual laboratory (MMVL)
\end{abstract}

\section{Background}

3D-Virtual Learning Environments (3D-VLEs) are the representation of 3D space inside the computer memory which enables students to navigate freely inside the virtual world and process different objects in real time[1, 2]. Adaptive 3D-Virtual Learning Environments (3D-VLEs) change its contents at run time according to the learning needs of individual student[3, 4]. These environments have many advantages. It display personalized learning contents to each student, reduces the probability of astray navigation inside the virtual environment and makes the distinction between education and entertainment which improve the learning process of students [5-7]. In literature a lot of work has been done which support the use of cognitive aids (Audio, Visual and textual etc) in virtual learning environments. For example, Nguyen et al.[8] used lighting source, arrows, and a compass as navigational guiding aids which help trainees to find the target. Gavish et al.[9] presented a 3D virtual puzzle in which verbal instructions plus mouse pointing are used to find the target location on learner screen. Similarly, Ali et al. [3] developed Multimodal Virtual laboratory (MMVL in which students were provided both audio and visual support during experiments which improved their efficiency.

The use of these aids reduce mental load on learner as it provide a specific and easy to follow instructions mechanism to perform the task [9]. However, these aids can act as shortcuts and prevents the trainees from active exploration which is necessary to perform the task independently in non-supervised environment [10-12]. Therefore, a mechanism is needed to use these aids while minimizing their negative effects. Jorge, R. et al.[13] recommended the control use of cognitive aids and proved that it will not affect the performance of the trainee in the real environment. Similarly, Rehman et al.[14], suggested the use of semantic aids with direct aids to maximize active exploration in virtual environments. In this paper we used adaptive repetition as a strategy to explore the 3D- Virtual Learning environments. The proposed approach enables the trainee to get the benefits of cognitive support while at the same time he is actively involved to explore the environment. We also tried to find the number of adaptive repetition required by the weak, average and good students to actively explore the virtual environment and perform the task successfully in non-supervised environment". The remaining paper is organized as below.

In section II we discussed the proposed approach followed by implementation in section III. In section IV we presented experimentations. Discussion is given in section V followed by conclusion in section VII.

\subsection{The Learning Decision Function (LDF)}

\section{The Proposed Approach}

With the help of Learning Decision Function (LDF), we can measure the learning skill of students in 3D-VLEs and declare it as weak, average and good learners [15]. The LDF takes time, no of errors and test scores of a student as inputs, calculate the learning skill of a student in the range of 0 to 1 and displays the result. If the function return value lies in the range [0, 0.3], the student is weak learner. If the return value lies in the range of $[0.3+\varepsilon, 0.6]$, the system consider the student as average learner. For good learner the return value exists in the range of $[0.6+\varepsilon, 0.1]$. 


\subsection{Adaptive Repetition}

In the initial stage the student is given Visual, audio, and textual support when he/she starts learning module $\mathrm{M}$ in 3D-VLEs. The system measure the total time taken by the students to complete the module, counts the number of errors he/she performs and calculates scores in the test given by the student at the end of the module. Using these three variables, the LDF declared the student as weak, average or good. Now to repeat the learning module $\mathrm{M}$, the system provides customized support for different learners. Weak students will follow path (X) which contains the same support of Visual, Audio, and textual aids. Average students will follow path (Y) for repetition which is backed up by visual and audio aids. Similarly, good students follow path (Z) which contains visual aid only or no support. In Figure 1 the proposed approach is presented graphically.

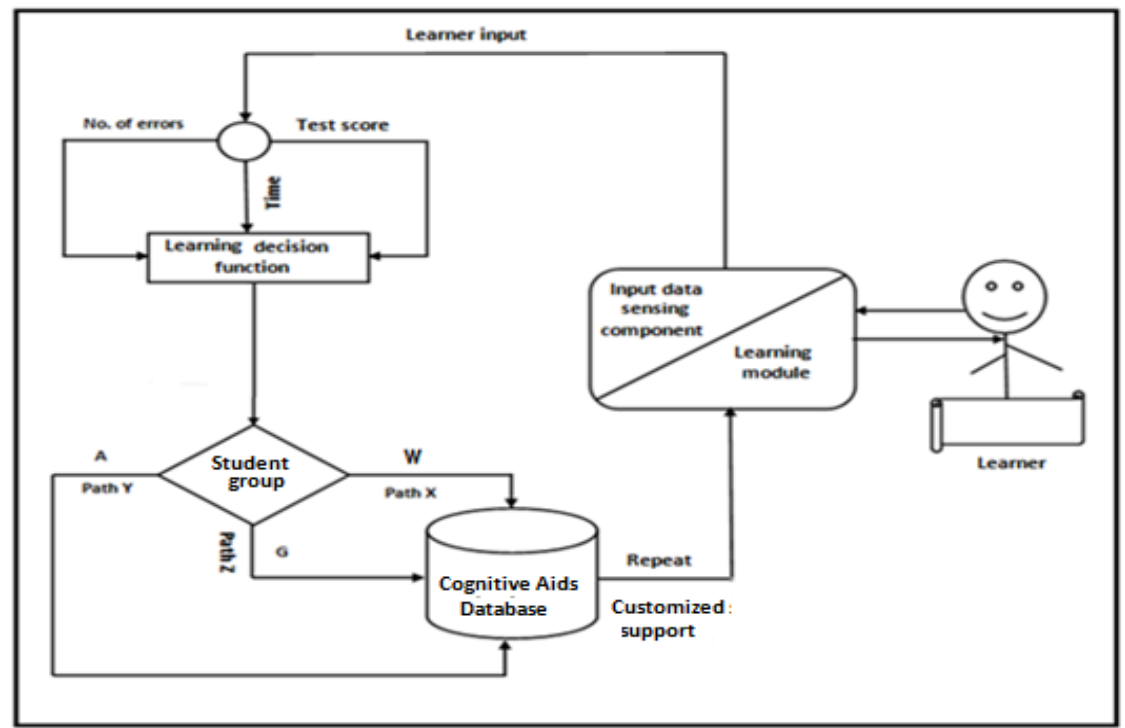

Figure 1 Adaptive Repetition

Path $(\mathrm{X})$ reduces cognitive load on students and is specially designed for weak learners. Path $(\mathrm{Y})$ facilitates average learners. In Path ( $\mathrm{Z}$ ), the cognitive support is small and it enforces the student to actively explore the environment during the learning process. It is designed for good learners.

\section{Implementation}

For the purpose of experimentation and analysis of the proposed approach we developed a Virtual Class room as shown in Figure 2. The environment was developed in MS Visual C++ 2008. OpenGL were used for rendering the graphics of the environment. Both Mouse and keyboard were used for interaction with the virtual environment. Simple Virtual Hand (SVH) is used for selection and manipulation of objects. The students get cognitive aids in the form of text, arrow and sound when he/she select objects within the environment. The students can see their performance in the environment on a board when they complete the learning module.

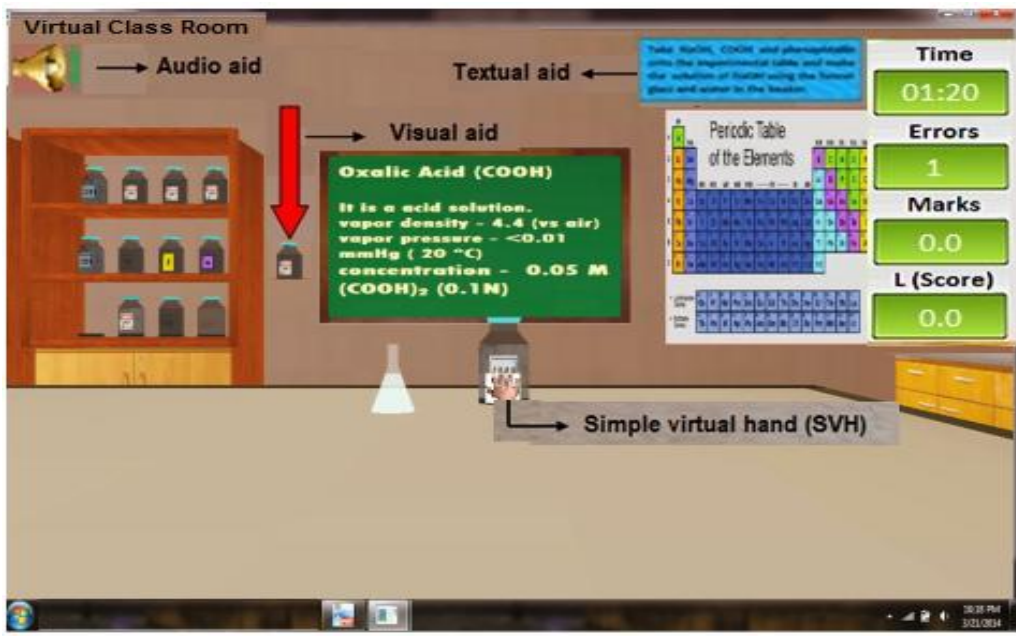

Figure 2 Virtual Class Room 


\section{Experimentations}

In order to perform experiments, we randomly selected 90 students both male and female of class 10th from 20 different schools and colleges. Based on their academic history and consultation with teachers, the students were divided into three groups as weak, average and good students. Students were taught how they will navigate and manipulate objects inside the virtual environment to perform their experiments. All students were asked to perform the experiment "To prepare $100 \mathrm{~cm} 3$ of $0.01 \mathrm{M}$ Oxalic Acid Solution from the given $0.1 \mathrm{M}$ Solution" repeatedly with the help of simulated environments. The process of repetition was stopped when a student was declared as good learner by the system and he/she maintains this capability in the next repetition. In Figure 3, two students are performing their experiments. The data was collected for weak, average and good students and is summarized as below.

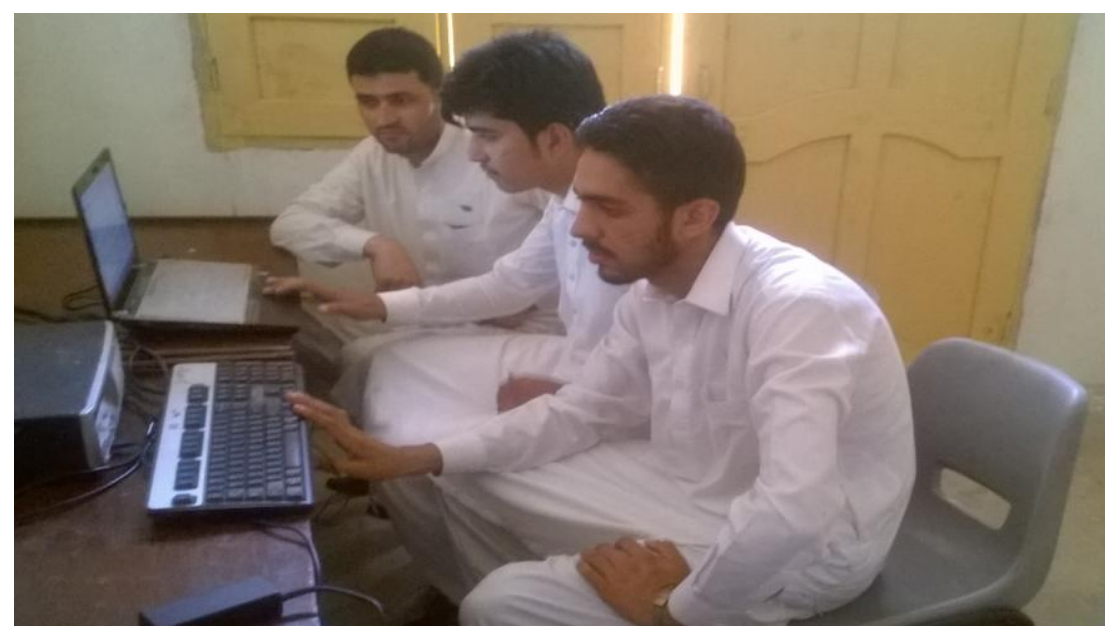

Figure 3 Students performing experiments

\subsection{Results and analysis}

\section{- Average No. of Adaptive Repetitions}

From the experimental data, it was observed that weak students performed 5 adaptive repetitions (on average) to actively explore the environment while average and good students performed 3 adaptive repetitions. These results are summarized in Figure 4 as below.

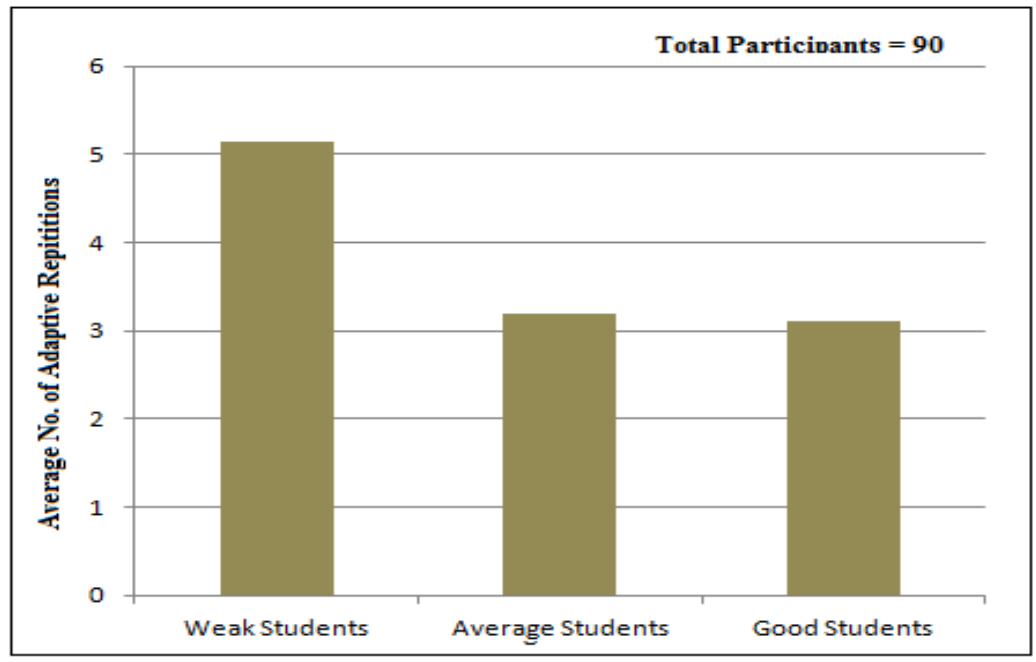

Figure 4 Average No. of Adaptive Repetitions

\section{- Average No. of Errors}

On average, Weak students performed 6 errors with standard deviation of 2.77 when they were repeating the experiments using the proposed approach. Average students performed 4 errors with standard deviation of 1.97. Similarly, 3 errors were performed by good students and their standard deviation was 0.91 . Figure 5 summarizes these results. 


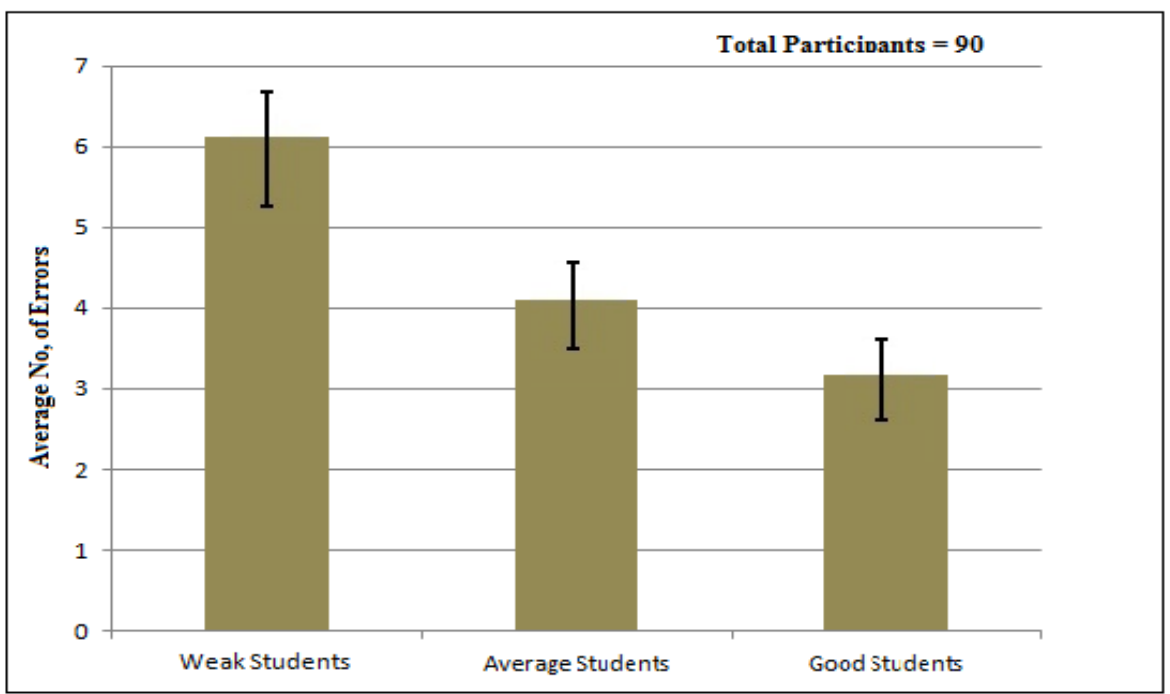

Figure 5 Average No. of Errors

\section{Discussion}

As mentioned before, the process of repetition was stopped when the student was declared as good learner by the system and he/she maintains this capability in the next repetition. Weak students performed 5 adaptive repetitions while average and good students performed 3 adaptive repetitions to fulfill the above criteria. In other words, students were able to perform the experiment without the help of cognitive aids. In their final repetitions, they were less dependent on aids and performed the task successfully. Below we clarify these statements.

The proposed approach gradually minimizes the support of aids and enforces the students to perform the task without the help of cognitive aids. In the initial stage the student is not familiar with the virtual environment. He/she performs more errors, take long time and get fewer score in the test. Therefore, the LDF value is small for him/her. He/she is considered weak student by the system and follows (path X) for repetition containing full support of aids which help him/her in performing their work. When the student repeats the learning module, his/her knowledge is increased about the environment and experiment. He/she gets high value of LDF which decreases the amount of aids for them. As a result students are forced to perform the work without the help of these aids which enable them to explore the environment.

\section{Conclusion}

In this study we proposed a strategy for active exploration of adaptive 3D-VLEs. The approach enables the students to use cognitive aids while at the same time he/she is actively involved in the learning process and exploration of the environment. From experimental results we concluded that weak students need 5 adaptive repetitions while average and good students need 3 adaptive repetitions to actively explore the environment and perform the task independently in non-supervised environment.

\section{References}

[1]. Ali, N., S. Ullah, and A. Alam, The Effect of Multimodalityand 3d Interaction In A Virtual Laboratory on Students'learning In Chemistry Education. Sindh University Research Journal-SURJ (Science Series),. 47(4), 2015.

[2]. $\quad$ Ali, N., et al., 3D Interactive Virtual Chemistry Laboratory for Simulation of High School Experiments. Paper presented at the Proceedings of EURASIA GRAPHICS 2014,Paper 12, 2014.

[3]. Alam, A., Ullah, S., Burqi, M. Z., Ullah, A., \& Ali, N. , Evaluating Students Performance in Adaptive 3D-Virtual Learning Environments Using Fuzzy Logic. Sindh University Research Journal-SURJ (Science Series), 48(2): p. 245-250, 2016.

[4]. Troyer, D., F.K. O., and A. Ewais, Enhancing virtual reality learning environments with adaptivity: lessons learned. in Symposium of the Austrian HCI and Usability Engineering Group, Springer., 2010.

[5]. Ewais, A. and O.D. Troyer, Authoring adaptive 3D virtual learning environments. International Journal of Virtual and Personal Learning Environments (IJVPLE). 5(1): p. 1-19, 2014.

[6]. Chittaro, L. and R. Ranon, Adaptive hypermedia techniques for 3D educational virtual environments. IEEE Intelligent Systems,. 22(4): p. 31-37, 2007.

[7]. $\quad$ Alam, A. and S. Ullah. Adaptive 3D-Virtual Learning Environments: From Students Learning Perspective. in FIT. 2016. Isalam Abad Pakistan: IEEE, p.7-10,2016.

[8]. Nguyen, T.T.H., T. Duval, and C. Fleury. Guiding techniques for collaborative exploration in multi-scale shared virtual environments. in GRAPP International Conference on Computer Graphics Theory and Applications. 2013.

[9]. Yuviler-Gavish, N., E. Yechiam, and A. Kallai, Learning in multimodal training: Visual guidance can be both appealing and disadvantageous in spatial tasks. International journal of human-computer studies,. 69(3): p. 113-122, 2011

[10]. Fu, W.-T. and W.D. Gray, Suboptimal tradeoffs in information seeking. Cognitive Psychology,. 52(3): p. 195-242, 2006.

[11]. Yechiam, E., I. Erev, and A. Parush, Easy first steps and their implication to the use of a mouse-based and a script-based strategy. 
Journal of Experimental Psychology: Applied,. 10(2): p. 89, 2004.

[12]. Craik, F.I. and R.S. Lockhart, Levels of processing: A framework for memory research. Journal of verbal learning and verbal behavior,. 11(6): p. 671-684, 1972.

[13]. Rodríguez, J., et al., Training of procedural tasks through the use of virtual reality and direct aids.: INTECH Open Access Publisher, 2012.

[14]. Rehman, I.U., S. Ullah, and I. Rabbi. The effect of semantic multi-modal aids using guided virtual assembly environment. in Open Source Systems and Technologies (ICOSST), 2014 International Conference on.. IEEE, 2014.

[15]. Alam, A., Sehatullah, and N. Ali, A Student-friendly Framework for Adaptive 3D-Virtual Learning Environments. Proceedings of the Pakistan Academy of Sciences: Pakistan Academy of Sciences A. Physical and Computational Sciences. 55(3): p. 255-266, 2016. 